
spécialité

\title{
Almut Koester, Workplace Discourse
}

Londres : Continuum, 2010

Philippe Millot

\section{(2) OpenEdition}

Journals

Édition électronique

URL : http://journals.openedition.org/asp/3799

DOI : 10.4000/asp.3799

ISSN : 2108-6354

Éditeur

Groupe d'étude et de recherche en anglais de spécialité

Édition imprimée

Date de publication : 1 novembre 2013

Pagination : 199-202

ISSN : 1246-8185

Référence électronique

Philippe Millot, «Almut Koester, Workplace Discourse », ASp [En ligne], 64 | 2013, mis en ligne le 01 novembre 2013, consulté le 04 novembre 2020. URL : http://journals.openedition.org/asp/3799; DOI : https://doi.org/10.4000/asp.3799

Ce document a été généré automatiquement le 4 novembre 2020.

Tous droits réservés 


\section{Almut Koester, Workplace Discourse}

Londres : Continuum, 2010

Philippe Millot

\section{RÉFÉRENCE}

Koester, Almut. 2010. Workplace Discourse. Londres : Continuum. 191 p. ISBN 978-1-8470-6115-7. 
1 Almut Koester, Professeur à l'Université de Birmingham et auteur de nombreux travaux sur les discours professionnels, propose, à partir d'une riche WORKPLACE DISCOURSE

ALMUT KOESTER bibliographie, une synthèse de plus de trente ans de recherche dans ce domaine, intitulée Workplace Discourse. Elle y présente également son travail, fondé sur le corpus d'anglais professionnel oral Corpus of American and British Office Talk (АВОТ), tout en accordant une place importante aux phénomènes plus récents tels que la communication électronique entre employés ou l'utilisation de l'anglais comme lingua franca. L'ouvrage comprend deux grandes parties. La première est consacrée à la définition du domaine (chapitre 1), aux différentes approches et méthodologies (chapitre 2) et aux caractéristiques principales du discours

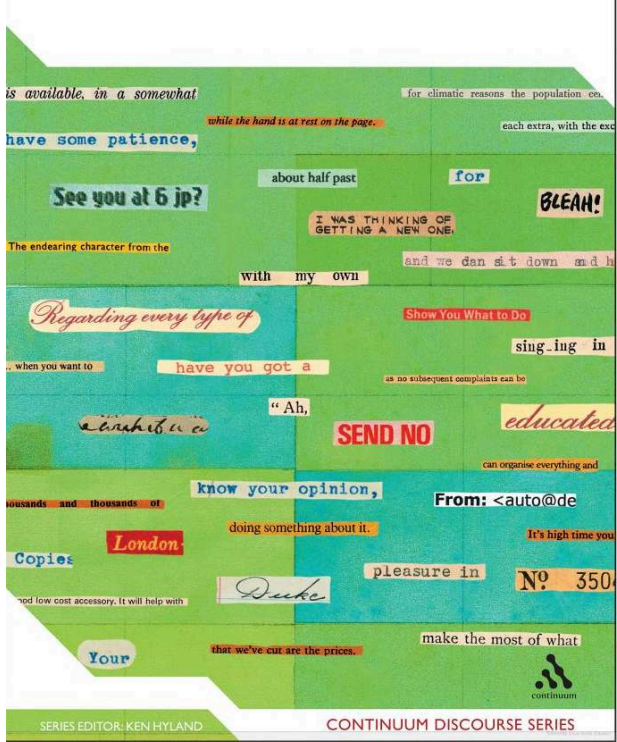
en milieu professionnel (chapitre 4). La

seconde partie traite de deux aspects très spécifiques mais répandus dans les échanges entre employés : l'informalité et les enjeux de l'augmentation de la communication internationale dans les entreprises depuis la fin du $\mathrm{XX}^{\mathrm{e}}$ siècle. L'ouvrage est complété par une annexe qui présente un extrait relativement long du corpus ABOT.

Dans le premier chapitre, l'auteur propose une réflexion épistémologique sur le discours en milieu professionnel, qu'elle envisage de manière large, comme un environnement spécialisé où diverses communautés, principalement composées d'experts, génèrent des discours qui leur sont propres. Ainsi, l'auteur distingue-t-elle le discours "des affaires»(business discourse), défini par l'orientation commerciale des échanges, du discours "professionnel », défini comme un discours réalisé entre experts, c'est-à-dire entre acteurs sociaux exerçant un métier particulier. Le troisième type de discours, le discours institutionnel, est quant à lui défini à travers l'ensemble des modes de fonctionnement qui régulent les échanges entre acteurs sociaux séparés par des différences d'ordre hiérarchique ou possédant des degrés d'expertise différents. Bien que les milieux professionnels soient tout à fait divers et les situations d'échange relativement hétéroclites, cette typologie permet à l'auteur de dessiner les contours d'un seul et même domaine de recherche, qui se présente d'emblée comme pluridisciplinaire et emprunte à des méthodes d'observation différentes, notamment l'observation participante, l'analyse de genre ou encore l'analyse de corpus. Outre ce travail de définition, l'auteur souligne que le discours en milieu professionnel tend à se complexifier à travers l'augmentation des échanges internationaux qui conduisent à la diversification des problématiques d'interface telles que les rapports interculturels en entreprise ou les échanges entre employés natifs et non natifs de l'anglais. Elle rappelle que ces processus tendent par ailleurs à s'accroître fortement depuis une vingtaine d'années, notamment à travers l'usage de technologies, telles que le courrier électronique, qui permettent l'échange rapide d'information à l'échelle de la planète. La 
fin de ce chapitre est consacrée à la distinction entre, d'une part, la zone des échanges de façade (frontstage talk), c'est-à-dire le discours au contact du public et, d'autre part, la zone des échanges réalisés dans les coulisses du monde professionnel (backstage talk). Parce que cette seconde zone d'échange implique généralement des experts entre eux, la dernière partie du chapitre invite clairement le chercheur en anglais de spécialité à l'explorer davantage pour en extraire les éléments spécialisés.

3 Dans le deuxième chapitre, l'auteur aborde la question de la spécificité du discours professionnel. En quoi un échange professionnel diffère-t-il d'un échange privé ou amical ? Selon l'auteur, la réponse tient à l'objectif utilitaire et spécialisé du discours donnant au genre un rôle central dans l'approche de cette question. L'auteur présente ainsi le genre très général de la résolution de problème comme un type de discours structurant d'autres genres récurrents dans le monde professionnel tels que la réunion, la prise de décision ou encore le courrier électronique. À partir d'une typologie très convaincante, l'auteur distingue ainsi les « genres textuels " (text-based genres), c'est-àdire des genres qui s'inscrivent dans une chaîne de textes, des " genres-action » (actionbased genres), que l'auteur présente comme des genres directement imbriqués dans l'action professionnelle en cours ou l'environnement physique des employés.

Le troisième chapitre aborde la question de la méthodologie et, plus particulièrement, celle du corpus comme méthode de caractérisation du discours en milieu professionnel. L'auteur commence par souligner la relative rareté des corpus du domaine liée à la difficulté d'accéder au milieu professionnel en général et à celui de l'entreprise privée en particulier. Elle poursuit en présentant trois outils d'analyse qui, selon elle, permettent à la fois de dégager la spécificité de certains domaines professionnels (spécialisés) et de contribuer à la caractérisation du discours professionnel en général : les mots clés, les collocations et les paquets lexicaux (chunks). Sur ce point, le lecteur peut noter que les exemples de collocations et de paquets lexicaux qu'elle donne aux pages 53 et 54 sont la preuve de l'existence d'une phraséologie professionnelle au même titre qu'il existe une phraséologie universitaire, c'est-à-dire des unités lexicogrammaticales de sens restreint et transversales à la plupart des domaines professionnels.

5 Dans les chapitres 4 et 5 , l'auteur présente les caractéristiques principales du discours en milieu professionnel à partir du corpus $\mathrm{ABOT}$, un corpus d'anglais professionnel qu'elle a personnellement conçu et qu'elle présente de manière détaillée dans son ouvrage précédent (Koester 2006). Le chapitre 4 traite de trois genres professionnels récurrents : le discours procédural, la requête et la prise de décision. À partir d'une analyse fine et approfondie, l'auteur montre comment et à quel point les instructions, comme exemple d'acte de parole typique de ces trois genres, présentent des variations de forme en fonction des domaines spécialisés et en fonction des genres eux-mêmes. Ainsi, l'auteur montre qu'en dépit du caractère mécanique que peuvent présenter certaines routines langagières professionnelles, lesquelles routines sont véhiculées de manière parfois caricaturale dans les manuels d'anglais professionnel, le discours en milieu professionnel se présente à l'observateur sous forme d'une équation relativement complexe. Dans cette équation, certains paramètres tels que le domaine spécialisé ou le rôle social et discursif qu'occupent les participants à l'échange peuvent donner lieu à des résultats contre-intuitifs jetant, pour ainsi dire, un certain trouble dans les genres professionnels. La dernière partie du chapitre 4 présente le thème 
encore peu exploré de la simulation narrative et du recours à l'imaginaire dans les séances de formation professionnelle.

6 Le chapitre 5 traite des échanges relationnels (relational talk) dont elle distingue deux types: les conversations informelles et routinières (small talk) et les conversations à caractère humoristique. Bien qu'ils puissent être considérés comme des types de discours marginaux par rapport à des genres déjà bien documentés comme la réunion ou la résolution de problème, les échanges relationnels forment, selon l'auteur, une caractéristique centrale et transversale aux milieux professionnels. Par exemple, dans la section consacrée à l'humour au travail, l'auteur montre que la fréquence des traits d'humour solidaire (supportive humour), bien qu'ils remplissent la fonction générale d'atténuation des tensions au travail, dépend fortement du type de communauté professionnelle, qu'elle soit une communauté de métier ou une entreprise.

7 Dans le chapitre 6, A. Koester aborde la question de l'anglais comme langue internationale d'échange. L'auteur rappelle que, certes, le phénomène est relativement ancien mais qu'il suscite depuis environ deux décennies une certaine controverse scientifique entre les défenseurs d'un modèle international de l'anglais, relativement traditionnel, c'est-à-dire calqué sur le modèle natif, et l'approche plus radicale des défenseurs de la variété « anglais lingua franca » (English as a Lingua Franca) fondée sur le renversement conceptuel d'une variété d'anglais non native. Une large part de ce chapitre est consacrée à cette variété émergente. L'auteur rappelle tout d'abord les fondements théoriques qu'elle résume comme le passage d'un modèle de la déficience par rapport au modèle natif à celui d'un modèle légitime, en voie de caractérisation. Elle présente ensuite les principales caractéristiques de l'anglais lingua franca en général et de l'anglais lingua franca des affaires (Business English as a Lingua Franca, BELF) en particulier. Selon l'auteur cependant, la stabilité de quelques caractéristiques, très générales au demeurant, ne sauraient masquer les contradictions qu'elle observe dans les résultats publiés en la matière. L'auteur pose alors un problème de taille, celui d'une variété si dépendante du contexte qu'elle freinerait tout effort de généralisation. Outre ce problème qui nous semble d'ordre principalement méthodologique, le chercheur en anglais de spécialité relèvera que la variété BELF, le pendant professionnel de l'anglais lingua franca, est présentée comme une variété intrinsèquement spécialisée en ce que les connaissances professionnelles et disciplinaires sont, dans bien des cas, l'un des rares points communs entre locuteurs de langues et de cultures nationales différentes.

8 Le chapitre 7 traite des applications de la recherche à des fins pédagogiques et de formation professionnelle. L'auteur observe d'abord un certain décalage entre les manuels d'anglais professionnel et l'anglais tel qu'il est pratiqué sur le terrain. Ce décalage viendrait du fait que ces manuels tendent à sous-estimer l'importance de la culture socioprofessionnelle dans l'apprentissage au profit de l'apprentissage de formules préfabriquées qui, comme le rappelle l'auteur, sont en réalité assez rares dans les corpus d'anglais professionnel. Elle observe ensuite que si les genres écrits, parce qu'ils sont solidement codifiés et relativement faciles à obtenir, occupent une large place dans les formations en anglais professionnel, les genres oraux, plus fluides et relativement difficiles à obtenir, restent les parents pauvres de ce type de formation. L'auteur s'appuie alors sur ses propres travaux pour dégager ce qui pourrait former la base théorique d'une pédagogie de l'anglais appliqué aux milieux professionnels. Notons que cette base est d'autant plus solide qu'elle s'appuie sur les six chapitres 
précédents ainsi que sur un nombre très élevé d'exemples de données naturelles tirés du corpus de l'auteur.

9 En conclusion, l'ouvrage dans son ensemble s'avère d'une double utilité. Il est tout d'abord utile à tout enseignant exerçant dans les branches professionnelles du domaine des langues pour spécialistes des autres disciplines, lesquels enseignants n'ont souvent d'autre choix que de recourir à des manuels d'anglais professionnel dont Almut Koester, sans les renier pour autant, sait montrer les limites. Ensuite, cet ouvrage présente une grande utilité aux chercheurs souhaitant soit s'initier au vaste domaine du discours en milieu professionnel, soit approfondir leur connaissance de certaines caractéristiques spécifiques de ce type de discours tels que les genres-actions ou les échanges informels entre employés.

\section{BIBLIOGRAPHIE}

Koester, Almut. 2006. Investigating Workplace Discourse. Londres : Routledge.

\section{AUTEURS}

PHILIPPE MILLOT

Université Grenoble Alpes, [ILCEA], F-38040 Grenoble 